\title{
An Evaluation of the Pharmacodynamics, Safety, and Tolerability of the
}

\section{Potassium Binder RDX7675}

Bertram Pitt, MD', Vanessa Zann, $\mathrm{PhD}^{2}$, Chris Roe, BSc${ }^{2}$, Jeffrey W. Jacobs, $\mathrm{PhD}^{3}$, James P.

Davidson, $\mathrm{PhD}^{3}$, Christine Dowd ${ }^{3}$, Padmapriya Kumaraswamy, MPharm ${ }^{3}$, Fangling Lin,

$\mathrm{MSc}^{3}$, Paul Korner, $\mathrm{MD}^{3}$, Robert C. Blanks, $\mathrm{MSc}^{3}$, and David P. Rosenbaum, $\mathrm{PhD}^{3}$

${ }^{1}$ Department of Medicine, University of Michigan School of Medicine, Ann Arbor, MI, USA

${ }^{2}$ Quotient Clinical, Ruddington, Nottingham, UK

${ }^{3}$ Ardelyx, Inc., Fremont, CA, USA

\section{Corresponding Author:}

David P. Rosenbaum, PhD, Ardelyx, Inc., 34175 Ardenwood Boulevard, Suite 200, Fremont,

CA 94555.Tel.: +1 510745 1752; Email: drosenbaum@ardelyx.com

\section{Acknowledgments}

The study was performed by Quotient Clinical, Nottingham, UK, and the Principal Investigator was Jo Collier, MBChB, FFPM, Dip Stats (OU). Medical writing support was provided by Richard Claes, $\mathrm{PhD}$, and Tim Ellison, PhD, of PharmaGenesis London, London,

UK, funded by Ardelyx, Inc. (Fremont, CA, USA).

\section{Declaration of Conflicting Interests}

Bertram Pitt is a consultant for Ardelyx, AstraZeneca, AuraSense, Bayer, Kwangdong Pharmaceuticals, Relypsa, Sanofi, scPharmaceuticals, Stealth Peptides, and Tricida, and has

This is the author manuscript accepted for publication and has undergone full peer review but has not been through the copyediting, typesetting, pagination and proofreading process, which may lead to differences between this version and the Version of Record. Please cite this article as doi: 10.1002/jcph.1102.

This article is protected by copyright. All rights reserved. 
ownership interest in AuraSense, Kwangdong Pharmaceuticals, Relypsa, scPharmaceuticals, and Tricida.

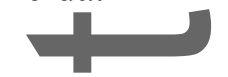

Vanessa Zann and Chris Roe are employees of Quotient Clinical.

JeffreyW. Jacobs, James P. Davidson, Padmapriya Kumaraswamy, Fangling Lin, Paul Korner, Robert C. Blanks, and David P. Rosenbaum are employees of Ardelyx, Inc. and have ownership interest in Ardelyx, Inc. Christine Dowd is a former employee of Ardelyx, Inc.

\section{Funding}

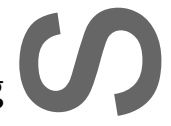

This study was funded by Ardelyx, Inc. (Fremont, CA, USA).

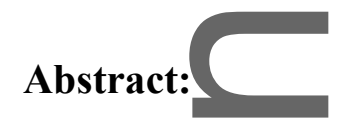

Hyperkalemia is common in patients with heart failure or chronic kidney disease, particularly those taking renin-angiotensin-aldosterone system inhibitors, and can cause arrhythmias and sudden cardiac death. The most widely used treatment, sodium polystyrene sulfonate (SPS), limits gastrointestinal potassium absorption, but has poor palatability. RDX7675 (RDX227675) is the calcium salt of a re-engineered polystyrene sulfonate-based resin, with improved palatability over SPS. The pharmacodynamic effects and safety of RDX7675 were assessed in a phase 1, single-center, randomized, active-controlled study. Healthy volunteers received nominal active doses of RDX7675 4.6 g b.i.d., 4.6 g t.i.d., 6.9 g b.i.d., 13.7 g q.d., 9.2 g t.i.d., or 13.7 g b.i.d. $(n=12$ each), or equivalent doses of SPS ( $n=3$ each), for 4 days. RDX7675 dosing increased stool potassium excretion and decreased urinary potassium excretion from baseline. Stool potassium excretion increased by up to $1481 \mathrm{mg} /$ day with RDX7675 (6.9 g b.i.d.), and urinary potassium excretion decreased by up to $939 \mathrm{mg} /$ day (13.7 g b.i.d.). Similar levels of potassium excretion were observed using q.d., b.i.d., or t.i.d. dosing of a $13.7 \mathrm{~g}$ total daily RDX7675 dose. Few adverse events were reported. In 
conclusion, repeated oral dosing with RDX7675 over 4 days reduced potassium absorption in healthy volunteers; the results support q.d. dosing of RDX7675 in future clinical studies.

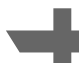

Keywords: RDX7675, RDX227675, sodium polystyrene sulfonate, potassium binder, pharmacodynamics, hyperkalemia

\section{Introduction}

Hyperkalemia, an elevated level of serum potassium, is a potentially life-threatening condition with consequences that can include cardiac arrhythmias and sudden cardiac death. ${ }^{1}$ The condition is often defined as a serum potassium concentration of at least 5.0 or $5.5 \mathrm{mmol} / \mathrm{L}$, and mortality risk increases with rising serum potassium concentrations above $5.0 \mathrm{mmol} / \mathrm{L}^{2-4}$ However, levels above these thresholds do not affect all patients in the same manner. 4,5 Only one half of patients with serum potassium above $6.5 \mathrm{mmol} / \mathrm{L}$ demonstrate electrocardiographic changes and there is no defined threshold above which hyperkalemia distinctly causes adverse events or death, because the risk depends in part on the rate of rise of serum potassium, as well as $\mathrm{pH}$, concomitant cardiovascular medications and comorbidities. ${ }^{4-6}$ Typical symptoms of hyperkalemia may include fatigue, muscle weakness, heart palpitations, nausea, vomiting, chest pain, and paresthesia. However, hyperkalemia is often asymptomatic, thus highlighting the importance of monitoring patients at high risk of developing the condition. ${ }^{7}$

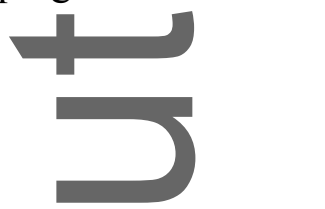

Hyperkalemia most commonly develops in patients older than 65 years and with comorbid conditions, such as chronic kidney disease (CKD), heart failure, or diabetes. ${ }^{3,4,8}$ In patients with CKD, hyperkalemia can occur through a decreased glomerular filtration rate and mild acidosis, which limit renal excretion of potassium. ${ }^{3,4}$ A cross-sectional study of pre-dialysis 
patients with advanced CKD reported a prevalence of hyperkalemia of $32 \%$ (serum potassium $\geq 5.5 \mathrm{mmol} / \mathrm{L}),{ }^{9}$ while a study monitoring patients undergoing maintenance dialysis found that $12.5 \%$ had a 3 -month-average serum potassium level above $5.5 \mathrm{mmol} / \mathrm{L}^{10}$ Treatmentguidelines for patients with CKD and heart failure recommend renin-angiotensinaldosterone system (RAAS) inhibitors as a first-line therapy to improve renal and cardiovascular outcomes and thus reduce morbidity and mortality. ${ }^{11-13}$ However, RAAS inhibitors decrease kidney excretion of potassium through their inhibitory effect on aldosterone, which can exacerbate hyperkalemia risk in patients with CKD, diabetes, and/or heart failure because of certain physiological disturbances associated with these conditions, such as aldosterone deficiency, decreased delivery of sodium to the distal nephron, and abnormal functioning of the cortical collecting tubule. ${ }^{14}$ Concern over the development of hyperkalemia in patients with CKD and/or heart failure can lead to RAAS inhibitor dose reduction and discontinuation, which increases cardiovascular risk. ${ }^{8,15}$ There is, therefore, a need for agents that can help manage or prevent hyperkalemia in the long term and thereby enable concomitant use of optimal RAAS inhibitor therapy.

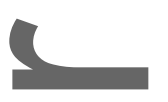

Orally administered

Orally administered cation exchange resins have been the dominant mode of treatment for acute or episodic hyperkalemia for more than 60 years. These compounds reduce potassium absorption by sequestering potassium in the gastrointestinal tract, thereby increasing potassium elimination in stool. Sodium polystyrene sulfonate (SPS; Kayexalate ${ }^{\circledR 16}$ or Resonium $A^{\circledR 17}$, the latter not marketed in the USA) is one of the most widely used potassium binders, and was first approved by the US Food and Drug Administration (FDA) in $1958 .{ }^{18}$ However, SPS has several limitations that make it unsuitable as a long-term therapy for hyperkalemia. First, the sodium counter-exchange ion of SPS increases the dietary sodium 
intake of patients taking this medication, which negatively impacts the RAAS ${ }^{19}$ and is believed to limit responsiveness to RAAS inhibition. ${ }^{20}$ Indeed, excessive sodium intake counteracts the dietary salt restriction recommended in patients with $\mathrm{CKD}$ and/or heart failure taking RAAS inhibitors. ${ }^{21,22}$ Secondly, SPS consists of hard, irregularly shaped, shard-like particles that give it a gritty texture in the mouth, which, together with its unpleasant taste, reduces palatability and is therefore likely to reduce adherence in a long-term setting. Individuals with CKD and/or heart failure are prescribed multiple medications to take at various times of the day, making palatability particularly important for facilitating long-term medication use. Thirdly, to alleviate its constipating effect, SPS is sometimes used in combination with sorbitol. In September 2009, the FDA recommended against the concomitant use of sorbitol with SPS as a result of reported cases of colonic necrosis and other severe gastrointestinal side effects (although such effects are rare). ${ }^{23}$ The precise mechanism is unknown; however, it has been postulated that sorbitol, rather than SPS, may be the cause. ${ }^{24}$ In addition to the aforementioned limitations, SPS was approved over 50 years ago and has not undergone testing in accordance with current regulatory standards, although it has been widely used in clinical studies. ${ }^{4}$

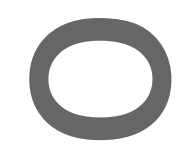

RDX7675 (Supplemental Figure S1), also known as RDX227675, is a novel potassiumbinding agent in development for the treatment of hyperkalemia. It is the calcium salt of a polystyrene sulfonate resin that has been re-engineered to improve potassium-binding efficiency compared with $\operatorname{SPS}^{25}$ and overcome many of the latter's undesirable aspects. For example, the RDX7675 resin comprises smooth, spherical, malleable beads with a relatively narrow size range ( $80 \%$ with diameter $14-52 \mu \mathrm{m}$; SPS, $11-124 \mu \mathrm{m}$; both measured using wet dispersion laser diffraction), giving it improved texture, taste, and overall palatability 
compared with the gritty shard-like particles of SPS. ${ }^{26}$ Indeed, a palatability study conducted in healthy volunteers showed that RDX7675 had a higher rate of overall acceptability in the mouth compared with SPS. ${ }^{26}$ RDX7675 may therefore be more acceptable to patients than SPS for long-term use, particularly given that the recommended dosage for SPS requires up to four daily administrations. ${ }^{16}$ Importantly, RDX7675 also has a calcium rather than sodium counter-exchange ion, so its administration does not increase dietary sodium intake.

Moreover, RDX7675 is not intended to be taken with sorbitol, thereby avoiding the potential for sorbitol-related gastrointestinal side effects. RDX7675 is insoluble in aqueous solutions and is not absorbed from the gastrointestinal tract, which also minimizes the potential for offtarget side effects. In preclinical studies in mice, RDX7675 administration has been found to reduce potassium absorption in a dose-dependent manner versus control animals. ${ }^{25}$

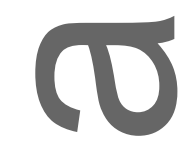

The aim of the phase 1, single-center, randomized, active-controlled study in healthy volunteers reported here was to investigate the pharmacodynamic effects and safety of different dosing regimens of RDX7675 in humans.

\section{Methods}

\section{Study Overview}

The study protocol and other documentation for this phase 1, single-center, randomized, active-controlled study, conducted at Quotient Clinical (Nottingham, UK), were approved by the Wales Research Ethics Committee (Cardiff, UK) before the study began. All volunteers provided written informed consent, and the study was conducted in accordance with International Conference on Harmonisation Good Clinical Practice guidelines and with the Declaration of Helsinki and its amendments. The study evaluated the relative safety and 
tolerability of RDX7675 compared with SPS, and determined the pharmacodynamics of different oral dose regimens of RDX7675.

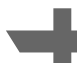

\section{Study Participants}

Healthy men and women aged $18-65$ years with a body mass index of $18-32 \mathrm{~kg} / \mathrm{m}^{2}$ were eligible toparticipate. From 24 hours before admission up until discharge from the study, volunteers were required to abstain from the following: alcohol; food likely to disturb gastrointestinal transit, such as spicy, high-fat, or high-fiber foods; and liquids or food containing grapefruit, cranberry, caffeine, or other xanthines. In addition, volunteers were required to abstain from food containing poppy seeds from 48 hours before admission until discharge from the study, and from unaccustomed strenuous exercise for 72 hours before the screening visit and then from 72 hours before admission until discharge from the study.

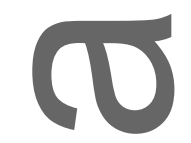

Key exclusion criteria were: a history of hypokalemia, hypomagnesia, or hypercalcemia; serum potassium below the lower limit of normal $(<3.5 \mathrm{mmol} / \mathrm{L})$ at baseline (day -2$)$; an allergy or history of sensitivity to SPS; use of treatments or supplements that are known to affect stool consistency or gastrointestinal motility, including fiber supplements, probiotic medications or supplements, antidiarrheals, prokinetic drugs, and enemas, in the week before admission; and use of salt or electrolyte supplements containing sodium, potassium, chloride, or bicarbonate formulations in the week before admission.

\section{Study Design}

Volunteers attended a screening visit up to 28 days before the first dose of study drug to confirm eligibility for the study. Eligible participants were admitted to the clinical unit in the evening 3 days before the first dose (day -3$)$. On the morning of day 1 , volunteers were randomly assigned using a computer-generated randomization schedule to 4 days of 
treatment with either RDX7675 or SPS in a 4:1 ratio, divided into cohorts with different dosing regimens to explore the relative efficacies of once-daily (q.d.), twice-daily (b.i.d.), and three times daily (t.i.d.) dosing (Table 1). The nominal active doses of each agent were determined following adjustment for counter-exchange ion content. Hereafter, unless otherwise stated, all regimens are given in terms of the nominal active dose, and data are presented imorder of increasing total daily dose rather than the order in which the regimens were evaluated.

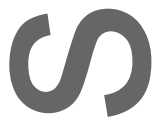

Within each cohort, volunteers received either a synthetic vanilla-flavored jelly-like formulation of RDX7675 (prepared in water thickened with 1\% xanthan gum) or the corresponding doses of water-reconstituted SPS (Resonium A, vanilla flavor) as an active comparator. The recommended starting dose of SPS is a mass of $15 \mathrm{~g}$ taken three or four times per day, ${ }^{16}$ and based on preclinical data ${ }^{25}$ it was anticipated that the therapeutic dose of RDX7675 would be similar. As this was the first swallowed dose of this RDX7675 formulation given to humans, a comparatively low starting dose of $4.6 \mathrm{~g}$ t.i.d. was selected. Following completion of dosing in each regimen group, there was an interim review of the available safety and pharmacodynamic data in order to select the dose level of RDX7675 and SPS for the next cohort. The maximum total daily dose of RDX7675 used in this study, 27.5 $\mathrm{g}$, was lower than the aforementioned recommended starting dose of SPS.

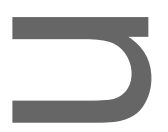

Volunteers were given their assigned dose of RDX7675 or SPS approximately 30 minutes after starting meals. Timings were approximately 08:00 for q.d. dosing, 08:00 and 18:00 for b.i.d. dosing, and 08:00, 13:00, and 18:00 for t.i.d. dosing. Discharge was on day 5, and a 
follow-up visit took place $2-5$ days later (day 7-10). Volunteers received the same meals/snacks in the same order on the same days while in the study center. Their diet was standardized for potassium content throughout the 4-day treatment period and the 2-day baseline period (i.e. days -1 and -2 ).

\section{Study Assessments}

Pharmacodynamic assessments included stool potassium, sodium, calcium, phosphorus, and magnesium content, and urinary potassium content, assessed over each 24-hour interval from day -2 to day 4. Time to first bowel movement, stool frequency, stool consistency (as measured by the Bristol Stool Form Scale [BSFS]), ${ }^{27}$ stool weight, and ease of passage were assessed from day -2 to day 4 . Safety assessments were performed at regular intervals and included physical examinations, clinical laboratory evaluations (hematology, clinical chemistry, urinalysis), vital signs, and electrocardiograms. Adverse events (AEs) were monitored throughout the study.

\section{Analytical Methods}

Electrolyte content of stool samples were determined by RTI International (Durham, NC, USA) using methodology previously described. ${ }^{28}$ The potassium content of urine samples was determined by The Doctors Laboratory (London, UK) using ion-selective electrode measurements obtained by standard clinical laboratory techniques (Consolidated Medical Bio-Analysis, Cypress, CA, USA).

\section{Statistical Analyses}

No formal statistical analysis was performed. For the pharmacodynamic assessments (electrolyte exeretion and stool parameters), descriptive statistics are presented. Baseline values for each analysis were calculated as the average of day -2 and day -1 . Only those participants who had sufficient urine and stool sampling data for pharmacodynamic parameter estimation were included in the pharmacodynamic population dataset. For the 
safety assessments, descriptive statistics are presented. All volunteers who received at least one dose of study medication were included in the safety analysis dataset.

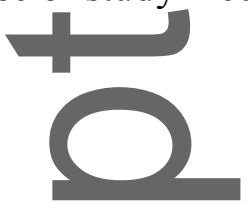

No formalsample size calculation was performed for this exploratory study. A sample size of ( 15 individuals per cohort (12 given investigational drug [RDX7675] and 3 given active comparator [SPS] is typical for phase 1 studies of this type and was expected to provide a sufficient number of observations to meet the objectives of the study.

\section{Results}

\section{Baseline Characteristics}

A total of 91 volunteers were enrolled into the six dose cohorts, of whom 90 completed the study (per cohort. RDX7675, $\mathrm{n}=12 ; \mathrm{SPS}, \mathrm{n}=3$ ). There were no notable differences in demographic characteristics between individuals assigned to RDX7675 or SPS (Table 2) or between the regimens. One volunteer in the 13.7 g q.d. cohort withdrew on day 1 having failed to swallow the given RDX7675 dose and with only baseline data recorded. This individual was included in the safety population, and a replacement volunteer was enrolled.

\section{Pharmacodynamics: Stool and Urinary Potassium Excretion}

Repeated dosing of RDX7675 or SPS over 4 days resulted in increased stool potassium excretion throughout the treatment period, with concomitant reductions in urinary potassium excretion. Mean increases in daily stool potassium from baseline were as high as $1481 \mathrm{mg}$ (6.9 g b.i.d.), with decreases in daily urinary potassium of up to $939 \mathrm{mg}$ (13.7 g b.i.d.) in the RDX7675 cohorts (Figure 1). Changes of a similar magnitude were generally observed in the SPS cohorts (Supplemental Figure S2). Stool potassium levels increased, and urinary potassium levels decreased, from baseline with increasing total daily RDX7675 dose over the 
4 day period, although this effect was less apparent after day 3 for the highest RDX7675 dose evaluated (27.5 g, i.e. 9.2 g t.i.d. and 13.7 g b.i.d.; Figure 1). Over 4 days, similar levels of potassium excretion were observed using q.d., b.i.d., and t.i.d. dosing of a $13.7 \mathrm{~g}$ total daily RDX7675-dose(Figure 1).

\section{Pharmacodynamics: Excretion of Other Electrolytes in Stool}

Excretion of other electrolytes in stool was highly variable, as expected. Volunteers taking RDX7675 generally had increased stool sodium and calcium excretion with no consistent effects on stool phosphorus or magnesium excretion (Table 3).

\section{Pharmacodynamics: Stool Parameters}

Data for stool frequency, stool consistency, ease of passage, and stool weight at baseline and on day 4 for volunteers who received RDX7675 are presented in Table 4. In general, on day 4 of treatment, there were increases from baseline in stool frequency and stool weight following dosing with RDX7675 or SPS. Mean baseline stool frequency in the RDX7675 cohorts ranged from 1.0 to 1.2 bowel movements/day, which increased to between 1.3 and 1.6 bowel movements/day after 4 days of dosing. Mean baseline stool weight with RDX7675 ranged from $70 \mathrm{~g} /$ day to $140 \mathrm{~g} /$ day, increasing to between $136 \mathrm{~g} /$ day and $324 \mathrm{~g} /$ day on day 4 . There were no overall trends in stool consistency (BSFS score) or ease of passage between baseline and day 4 with either RDX7675 or SPS. Mean BSFS score with RDX7675 ranged from 2.7 to 4.0 at baseline, and from 2.3 to 4.6 on day 4 . Ease of passage with RDX7675 ranged between 3 (straining needed) and 4 (normal) throughout the monitoring period.

\section{Safety}

Both RDX7675 and SPS were generally well tolerated during repeated dosing for 4 days, with few AEs reported across all dosing regimens. The proportion of volunteers reporting AEs was similar with RDX7675 and SPS, with 24 of 73 and 6 of 18 volunteers reporting at least one AE after dosing with RDX7675 or SPS, respectively (Table 5). The number of 
volunteers reporting at least one AE was highest following dosing with RDX7675 9.2 g t.i.d. (10/12), $13.7 \mathrm{~g}$ b.i.d. (5/12), and $4.6 \mathrm{~g}$ t.i.d. (4/12). There was no apparent difference in the (3. incidence of AEs between the other RDX7675 regimens, or between the different SPS regimens. The most eommon AEs were gastrointestinal in nature, most frequently constipation, abdominal pain, and nausea. Although most AEs were mild in severity, a small number of AEs were of moderate intensity. Of the six moderate AEs experienced by volunteers receiving RDX7675, four were gastrointestinal in nature (nausea, constipation, and lower abdominal pain), all in the RDX7675 $9.2 \mathrm{~g}$ t.i.d. cohort. The most frequently reported treatment-related AEs were also gastrointestinal in nature (Table 5). The highest incidence of these treatment-related AEs occurred at the highest RDX7675 dose levels, $9.2 \mathrm{~g}$ t.i.d. and $13.7 \mathrm{~g}$ b.i.d., while there were no treatment-related AEs in the RDX7675 $6.9 \mathrm{~g}$ b.i.d. or $13.7 \mathrm{~g}$ q.d. cohorts. There were no serious or severe AEs during the study; however, one volunteer in the RDX7675 9.2 g t.i.d. cohort had treatment withdrawn as a result of moderate nausea (considered to be possibly treatment-related). There were no clinically significant findings in clinical laboratory assessments, including serum potassium, sodium, calcium, chloride or bicarbonate (Supplemental Table S1), as well as vital signs, electrocardiograms, or physical examinations.

\section{Discussion}

Treatment guidelines for patients with CKD and/or heart failure recommend the use of RAAS inhibitors, ${ }^{11-13}$ which can increase the risk of hyperkalemia. ${ }^{3}$ There is, therefore, a need for hyperkalemia therapies that can be used concomitantly with RAAS inhibitors over extended periods of time. Cation exchange resins that reduce potassium absorption from the gut have been used to treat hyperkalemia since the 1950s, with SPS being the most widely used of these medications. However, poor palatability and the potential for gastrointestinal side 
effects have limited the use of SPS as a long-term treatment. ${ }^{8,18}$ In recent years, new treatments for patients with hyperkalemia have been developed. Patiromer (Veltassa ${ }^{\circledR}$ ), approved for use in the USA and Europe, reduced serum potassium levels in short-term clinical trials in patients with hyperkalemia and CKD or heart failure, ${ }^{29,30}$ and in a long-term study in patients with hyperkalemia and diabetic nephropathy. ${ }^{30}$ Sodium zirconium cyclosilicate (ZS-9), which exchanges potassium for sodium ions, has been shown in two large phase 3 trials to lower serum potassium levels in a broad cross-section of patients with hyperkalemia. ${ }^{1,32}$ Patiromer has been associated with gastrointestinal adverse effects, including constipation and diarrhea, while ZS-9 has been associated with urinary tract infections and edema. ${ }^{33}$

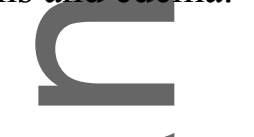

RDX7675, the ealcium salt of a re-engineered polystyrene sulfonate-based resin, is a (1) potassium binding agent that is being developed as a treatment for patients with hyperkalemia. Its novel design and formulation result in smooth, spherical, malleable beads with a relatively narrow size range (80\% with diameter $14-52 \mu \mathrm{m}$; SPS, $11-124 \mu \mathrm{m})$ for enhanced mouth-feel and palatability, shown in a healthy volunteer study to have improved acceptability compared with SPS. ${ }^{26}$ The use of calcium rather than sodium as the counterexchange ion, and administration without sorbitol or other cathartics, aligns with best clinical practice. ${ }^{22,23}$ The aim of the present study was to generate clinical data on the ability of several dosing regimens of RDX7675 to reduce potassium absorption, and their safety and tolerability, in healthy volunteers. 
In this study, repeated oral dosing with RDX7675 q.d., b.i.d., or t.i.d. over 4 days reduced potassium absorption, as shown by increases from baseline in stool potassium excretion with concomitant decreases in urinary potassium. The increases in daily stool potassium excretion observed after 4 days of RDX7675 administration (844-1481 mg in volunteers given total daily doses of 9.2-13.7 g) were of similar magnitude to those observed in a healthy volunteer study of patiromer (617-1929 mg in volunteers given total daily doses of 12.6-50.4 g [4.2$16.8 \mathrm{~g}$ t.i.d]). ${ }^{34}$ Although stool potassium levels generally increased and urinary potassium levels decreased with increasing total daily RDX7675 dose over the 4-day period, a wider RDX7675 dose range and/or a larger number of participants would likely be required to establish a dose-response relationship in healthy volunteers. Similar levels of potassium excretion were observed using q.d., b.i.d., and t.i.d. dosing of the same $13.7 \mathrm{~g}$ total daily RDX7675 dose, suggesting that q.d. dosing of RDX7675 will be appropriate for future clinical studies

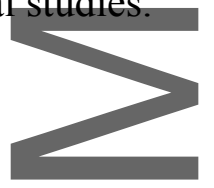

Stool sodium and calcium excretion generally increased from baseline with RDX7675 across the dosing regimens, in line with a propensity of polystyrene sulfonate to bind sodium and calcium; there were no consistent trends in stool magnesium or phosphorus excretion. In general, there were increases from baseline in stool frequency and stool weight following dosing with RDX7675, and no overall trends in stool consistency or ease of passage.

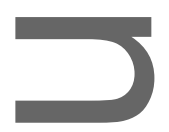

RDX7675 was generally well tolerated during repeated dosing over 4 days, with a low incidence of AEs. The most frequently reported AEs judged by the study investigator to be related to treatment were gastrointestinal in nature, most commonly abdominal pain and 
constipation. The incidence of these treatment-related AEs was highest at the highest RDX7675 doses, with few treatment-related AEs in the lowest dose cohorts. There were no serious or severe AEs.

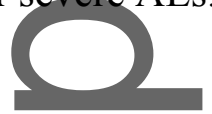

(1)

This study was not designed to compare the pharmacodynamic effects of RDX7675 and SPS, and the small number of volunteers given SPS (three per dose cohort) limits the power of any comparisons between RDX7675 and SPS. However, it was considered important to assess the relative safety and tolerability of the two compounds. The study was conducted open-label as the formulations of RDX7675 and SPS used had very different consistencies, and hence it was not practical to blind volunteers to study drug assignment.

\section{(U)}

The efficacy and safety data obtained from this study, together with the results of a palatability study showing improved acceptability of various oral formulations of RDX7675 over SPS, ${ }^{26}$ warrant the continued development of RDX7675 as a q.d. treatment for patients with hyperkalemia. Q.d. dosing, being more convenient for patients than taking medication multiple times per day, can be expected to result in improved compliance with RDX7675 compared with b.i.d. or t.i.d. regimens. A phase 3 clinical trial is planned, enrolling patients taking RAAS inhibitors and/or mineralocorticoid receptor antagonists and evaluating RDX7675 $10 \mathrm{~g}$ q.d. as a starting dose, similar to the q.d. dose investigated in the present phase 1 study. A trial evaluating the time to onset of action of RDX7675 in patients with hyperkatemia, together with safety and efficacy, is also planned, which will assess $10 \mathrm{~g}, 20 \mathrm{~g}$, and $30 \mathrm{~g}$ q.d. doses. This is a similar total daily dose range to that investigated in the present study, which was generally well tolerated. It is hoped that RDX7675 will address the unmet 
needs of patients with chronic hyperkalemia by proving to be an effective and well-tolerated therapy that is suitable for long-term use.

\section{Conclusions}

In healthy volunteers, repeated oral dosing with RDX7675 over 4 days reduced absorption of potassium, as shown by increases in stool potassium with concomitant reductions in urinary potassium Similar levels of potassium excretion were observed using q.d., b.i.d., and t.i.d. dosing of a 13.7 g total daily RDX7675 dose, supporting q.d. dosing in future clinical studies. RDX7675 was generally well tolerated, with few AEs reported. These data support the further clinical development of RDX7675 as a novel treatment for patients with hyperkalemia.

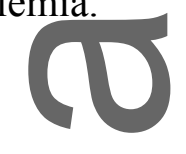

\section{References}

1. Evans KJ, Greenberg A. Hyperkalemia: a review. J Intensive Care Med.

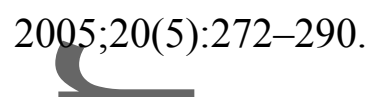

2. Jain N, Kotla S, Little BB, et al. Predictors of hyperkalemia and death in patients with cardiac and renal disease. Am J Cardiol. 2012;109(10):1510-1513.

3. Einhorn LM, Zhan M, Hsu VD, et al. The frequency of hyperkalemia and its significance in chronic kidney disease. Arch Intern Med. 2009;169(12):1156-1162.

4. Kovesdy CP. Management of hyperkalemia: an update for the internist. Am J Med. 2015; $128(12): 1281-1287$.

5. Welch A, Maroz N, Wingo CS. Hyperkalemia: getting to the heart of the matter. Nephrol Dial Transplant. 2013;28(1):15-16. 
6. Aronson PS, Giebisch G. Effects of $\mathrm{pH}$ on potassium: new explanations for old observations. J Am Soc Nephrol. 2011;22(11):1981-1989.

7. Lehnhard A, Kemper MJ. Pathogenesis, diagnosis and management of hyperkalemia. Pediatr Nephrol. 2011;26(3):377-384.

8. Pitt B, Bakris GL. New potassium binders for the treatment of hyperkalemia: current data and opportunities for the future. Hypertension. 2015;66(4):731-738.

9. Sarafidis PA, Blacklock R, Wood E, et al. Prevalence and factors associated with hyperkalemia in predialysis patients followed in a low-clearance clinic. Clin J Am Soc Nephrol. 2012;7(8):1234-1241.

10. Kovesdy CP, Regidor DL, Mehrotra R, et al. Serum and dialysate potassium concentrations and survival in hemodialysis patients. Clin J Am Soc Nephrol. 2007:2(5):999-1007.

11. KDIGO Clinical practice guideline for the management of blood pressure in chronic kidney disease. Kidney Int Suppl. 2012;2(5):347-356.

12. Yancy CW, Jessup M, Bozkurt B, et al. 2016 ACC/AHA/HFSA focused update on new pharmacological therapy for heart failure: an update of the 2013 ACCF/AHA guideline for the management of heart failure: a report of the American College of Cardiology/American Heart Association Task Force on clinical practice guidelines and the Heart Failure Society of America. J Am Coll Cardiol. 2016;68(13):1476-

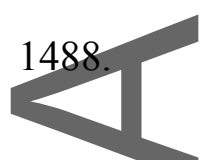

13. Yancy CW, Jessup M, Bozkurt B, et al. 2013 ACCF/AHA guideline for the management of heart failure: executive summary: a report of the American College of 
Cardiology Foundation/American Heart Association Task Force on practice guidelines. Circulation. 2013;128(16):1810-1852.

14. Palmer BF. Managing hyperkalemia caused by inhibitors of the renin-angiotensinaldosterone system. $N$ Engl J Med. 2004;351(6):585-592.

15. Dunn JD, Benton WW, Orozco-Torrentera E, Adamson RT. The burden of hyperkalemia in patients with cardiovascular and renal disease. Am J Manag Care. 2015;21(15 Suppl):s307-315.

16. Sanofi-Ayentis U.S. Kayexalate ${ }^{\circledR}$ (sodium polystyrene sulfonate [USP] cationexchange resin). Prescribing Information (2010). https://www.accessdata.fda.gov/drugsatfda_docs/label/2011/011287s0231bl.pdf. Accessed May 4, 2017.

17. Samofi-Aventis UK. Resonium $A^{\circledR}$ (sodium polystyrene sulfonate). Summary of Prochuct Characteristics (2014). https://www.medicines.org.uk/emc/medicine/6988. Accessed May 4, 2017.

18. Sterns RH, Rojas M, Bernstein P, Chennupati S. Ion-exchange resins for the treatment of hyperkalemia: are they safe and effective? J Am Soc Nephrol. 2010;21(5):733-735.

19. Williams JS, Williams GH, Jeunemaitre X, Hopkins PN, Conlin PR. Influence of dietary sodium on the renin-angiotensin-aldosterone system and prevalence of left ventricular hypertrophy by EKG criteria. J Hum Hypertens. 2005;19(2):133-138.

20. Krikken JA, Laverman GD, Navis G. Benefits of dietary sodium restriction in the management of chronic kidney disease. Curr Opin Nephrol Hypertens. 2009;18(6):531-538. 
21. Gupta D, Georgiopoulou VV, Kalogeropoulos AP, et al. Dietary sodium intake in heart failure. Circulation. 2012;126(4):479-485.

22. National Kidney Foundation Kidney Disease Outcomes Quality Initiative (NKF KDOQI). Clinical Practice Guidelines on Hypertension and Antihypertensive Agents in Chronic Kidney Disease.

https://wyw2.kidney.org/professionals/kdoqi/guidelines_bp/guide_6.htm. Accessed May 4,2017.

23. US Food and Drug Administration: Kayexalate (sodium polystyrene sulfonate) powder. Safety labeling changes approved by FDA Center for Drug Evaluation and Research (CDER) - September 2009.

http://www.fda.gov/Safety/MedWatch/SafetyInformation/ucm186845.htm. Accessed January 20, 2017.

24. Harel Z,Harel S, Shah PS, Wald R, Perl J, Bell CM. Gastrointestinal adverse events with sodium polystyrene sulfonate (Kayexalate) use: a systematic review. Am J Med. 2013;126(3):264 e269-224.

25. Davidson JP, King AJ, Kumaraswamy P, et al. Evaluation of the pharmacodynamic effects of the potassium binder RDX7675 in mice. J Cardiovasc Pharmacol Ther. 2017:1074248417741685.

26. Zann V, McDermott J, Jacobs JW, et al. Palatability and physical properties of potassium-binding resin RDX7675: comparison with sodium polystyrene sulfonate. Drug Des Devel Ther. 2017;11:2663-2673.

27. Lewis SJ, Heaton KW. Stool form scale as a useful guide to intestinal transit time. Scand J Gastroenterol. 1997;32(9):920-924. 
28. Johansson S, Leonsson-Zachrisson M, Knutsson M, et al. Preclinical and healthy volunteer studies of potential drug-drug interactions between tenapanor and phosphate binders. Clin Pharmacol Drug Dev. 2016:doi: 10.1002/cpdd.1307.

29. Pitt B, Anker SD, Bushinsky DA, Kitzman DW, Zannad F, Huang IZ. Evaluation of the efficacy and safety of RLY5016, a polymeric potassium binder, in a double-blind, placebo-controlled study in patients with chronic heart failure (the PEARL-HF) trial. Eur Heart J. 2011;32(7):820-828.

30. Weir MR,Bakris GL, Bushinsky DA, et al. Patiromer in patients with kidney disease and hyperkalemia receiving RAAS inhibitors. $N$ Engl J Med. 2015;372(3):211-221.

31. Kosiborod M, Rasmussen HS, Lavin P, et al. Effect of sodium zirconium cyclosilicate on potassium lowering for 28 days among outpatients with hyperkalemia: the HARMONIZE randomized clinical trial. JAMA. 2014;312(21):2223-2233.

32. Packham DK, Rasmussen HS, Lavin PT, et al. Sodium zirconium cyclosilicate in hyperkalemia. N Engl J Med. 2015;372(3):222-231.

33. Meaney CJ, Beccari MV, Yang Y, Zhao J. Systematic review and meta-analysis of patiromer and sodium zirconium cyclosilicate: a new armamentarium for the treatment of hyperkalemia. Pharmacotherapy. 2017:doi: 10.1002/phar.1906.

34. Li L, Harrison SD, Cope MJ, et al. Mechanism of action and pharmacology of patiromer, a nonabsorbed cross-linked polymer that lowers serum potassium coneentration in patients with hyperkalemia. J Cardiovasc Pharmacol Ther. $2016,21(5): 456-465$. 


\section{Supporting Information}

Additional supporting information may be found in the online version of this article at the publisher's website.

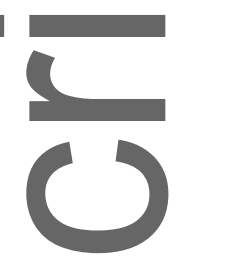

Tables

Table 1. Dosing Regimens of RDX7675 and SPS in the Healthy Volunteer Study

\begin{tabular}{|c|c|c|c|c|c|c|}
\hline $\begin{array}{l}\text { RDX7675/SPS } \\
\text { regimen }\end{array}$ & 5 g b.i.d. & 5 g t.i.d. & 7.5 g b.i.d. & 15 g q.d. & 10 g t.i.d. & 15 g b.i.d. \\
\hline $\begin{array}{l}\text { Nominal RDX7675 } \\
\text { active dose }\end{array}$ & 4.6 g b.i.d. & 4.6 g t.i.d. & 6.9 g b.i.d. & 13.7 g q.d. & 9.2 g t.i.d. & 13.7 g b.i.d. \\
\hline $\begin{array}{l}\text { Total daily } \\
\text { RDX7675 active } \\
\text { dose }\end{array}$ & $9.2 \mathrm{~g}$ & $13.7 \mathrm{~g}$ & $13.7 \mathrm{~g}$ & $13.7 \mathrm{~g}$ & $27.5 \mathrm{~g}$ & $27.5 \mathrm{~g}$ \\
\hline $\begin{array}{l}\text { Nominal SPS } \\
\text { active dose }\end{array}$ & $4.4 \mathrm{~g}$ b.i.d. & $4.4 \mathrm{~g}$ t.i.d. & $6.7 \mathrm{~g}$ b.i.d. & $13.3 \mathrm{~g} \mathrm{q.d.}$ & $8.9 \mathrm{~g}$ t.i.d. & $13.3 \mathrm{~g}$ b.i.d. \\
\hline $\begin{array}{c}\text { Total daily SPS } \\
\text { active dose }\end{array}$ & $8.9 \mathrm{~g}$ & $13.3 \mathrm{~g}$ & $13.3 \mathrm{~g}$ & $13.3 \mathrm{~g}$ & $26.7 \mathrm{~g}$ & $26.7 \mathrm{~g}$ \\
\hline $\begin{array}{l}\text { Cohort order of } \\
\text { enrollment }\end{array}$ & 4 & 1 & 3 & 5 & 2 & 6 \\
\hline
\end{tabular}

${ }^{\mathrm{a}}$ Mass of agent taken.

${ }^{\mathrm{b}}$ Active dose administered (after adjustment for counter-exchange ion content).

b.i.d., twice daily; q.d., once daily; SPS, sodium polystyrene sulfonate; t.i.d., three times daily. 
Table 2. Demographics and Baseline Characteristics of the Study Volunteers

\begin{tabular}{|l|c|c|}
\hline & $\begin{array}{c}\text { RDX7675 } \\
(\mathrm{N}=73)\end{array}$ & $\begin{array}{c}\text { SPS } \\
(\mathrm{N}=18)\end{array}$ \\
\hline Age, years & $36.5(13.5)$ & $39.4(14.9)$ \\
\hline Height, cm & $170.2(9.7)$ & $171.9(8.0)$ \\
\hline Weight, $\mathrm{kg}$ & $73.1(12.7)$ & $75.5(10.9)$ \\
\hline BMI, kg/m & $25.1(3.4)$ & $25.5(3.0)$ \\
\hline White, $\mathrm{n}(\%)$ & $65(89.0)$ & $14(77.8)$ \\
\hline Male, $\mathrm{n}(\%)$ & $35(47.9)$ & $10(55.6)$ \\
\hline
\end{tabular}

Data are mean (standard deviation) unless otherwise stated.

BMI, body massindex; SPS, sodium polystyrene sulfonate.

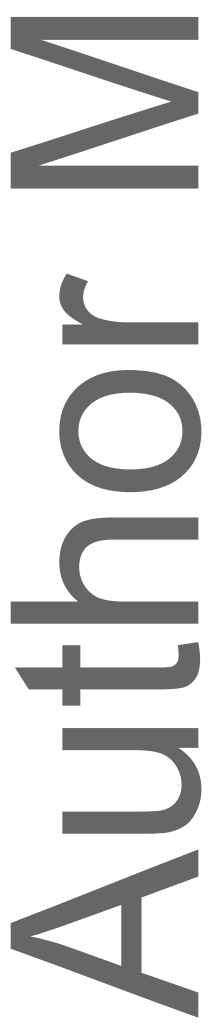


Table 3. Daily Excretion of Non-Potassium Electrolytes in Stool in Healthy Volunteers Given RDX7675 for 4 Days

\begin{tabular}{|l|c|c|c|c|c|c|}
\hline & \multicolumn{5}{|c|}{ RDX7675 } \\
\cline { 2 - 7 } & 4.6 g b.i.d. & 4.6 g t.i.d. & 6.9 g b.i.d. & 13.7 g q.d. & 9.2 g t.i.d. & 13.7 g b.i.d. \\
\hline & $(\mathrm{n}=12)$ & $(\mathrm{n}=12)$ & $(\mathrm{n}=12)$ & $(\mathrm{n}=12)$ & $(\mathrm{n}=12)$ & $(\mathrm{n}=12)$ \\
\hline Sodium, mg & & & & & \\
\hline Baseline & $24(43)$ & $44(50)$ & $24(18)$ & $13(14)$ & $16(16)$ & $20(23)$ \\
\hline Day 4 & $65(86)$ & $315(297)$ & $152(126)$ & $111(73)$ & $318(268)$ & $349(378)$ \\
\hline Calcium, mg & & & & & & \\
\hline Baseline & $239(288)$ & $407(187)$ & $423(242)$ & $314(219)$ & $331(201)$ & $331(242)$ \\
\hline Day 4 & $625(447)$ & $1093(585)$ & $979(338)$ & $879(400)$ & $726(454)$ & $770(626)$ \\
\hline Phosphorus, mg & & & & & & \\
\hline Baseline & $335(384)$ & $532(246)$ & $604(312)$ & $389(280)$ & $502(308)$ & $479(307)$ \\
\hline Day 4 & $606(460)$ & $805(435)$ & $792(283)$ & $644(375)$ & $370(238)$ & $451(404)$ \\
\hline Magnesium, mg & & & & & & \\
\hline Baseline & $56(56)$ & $96(47)$ & $109(55)$ & $69(54)$ & $85(57)$ & $77(47)$ \\
\hline Day 4 & $102(77)$ & $133(70)$ & $128(49)$ & $113(58)$ & $52(34)$ & $62(58)$ \\
\hline Dafa & & & & & & \\
\hline
\end{tabular}

Data are mean (standard deviation).

Baseline values are the mean for days -2 and -1 .

b.i.d., twice daily; q.d., once daily; t.i.d., three times daily.

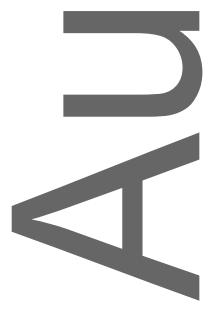


Table 4. Daily Stool Parameters of Healthy Volunteers Given RDX7675 for 4 Days

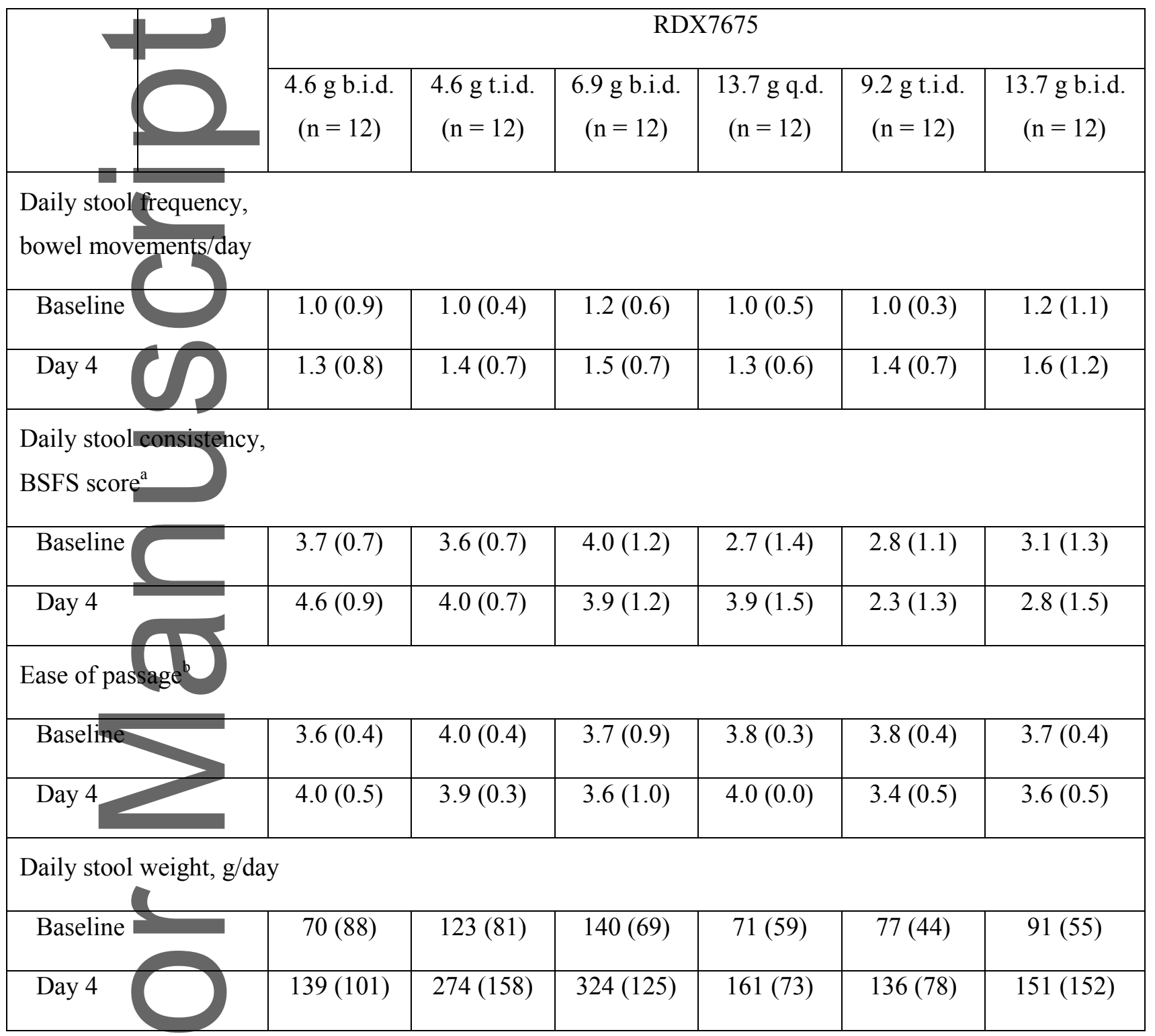

Data are mean (standard deviation). Baseline values are the mean for days -2 and -1 .

${ }^{a}$ BSFS: 1 =hard lumps; 2 = lumpy sausage; 3 = cracked sausage; 4 = smooth sausage; 5 = soft lumps;

$6=$ mushy; 7 = watery.

${ }^{\mathrm{b}}$ Ease of passage categories: 1 = manual disimpaction; 2 = enema needed; 3 = straining needed; $4=$ normal; $5=$ urgent without pain; $6=$ urgent with pain; $7=$ incontinent.

b.i.d., twice daily; BSFS, Bristol Stool Form Scale; q.d., once daily; t.i.d., three times daily. 
Table 5. Summary of Adverse Events in Healthy Volunteers Given RDX7675 or SPS for 4

Days

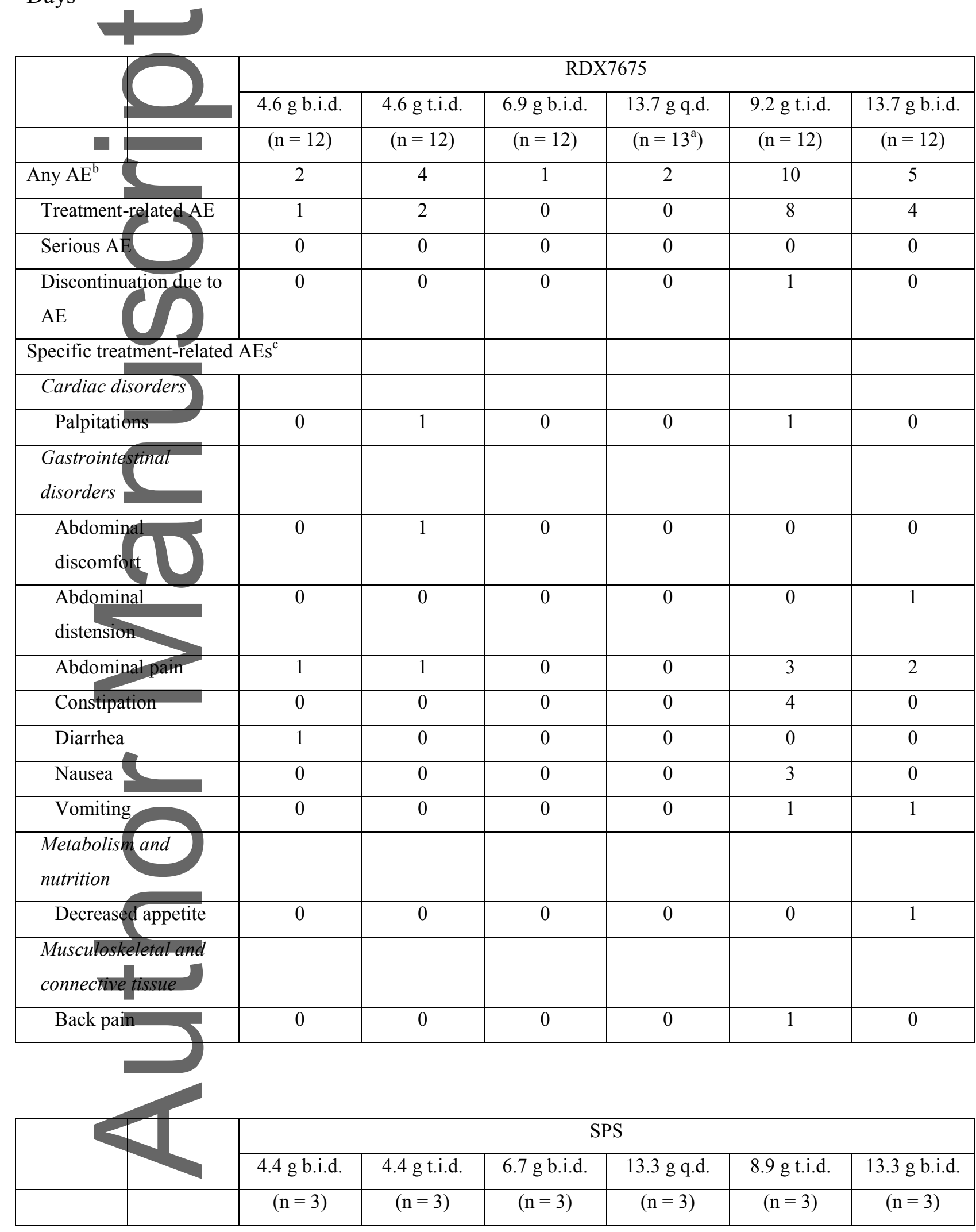




\begin{tabular}{|c|c|c|c|c|c|c|}
\hline Any $\mathrm{AE}^{\mathrm{b}}$ & 1 & 0 & 2 & 1 & 1 & 1 \\
\hline Treatment-related AE & 0 & 0 & 1 & 0 & 1 & 1 \\
\hline Serious AE & 0 & 0 & 0 & 0 & 0 & 0 \\
\hline $\begin{array}{l}\text { Discontinuation due to } \\
\mathrm{AE}\end{array}$ & 0 & 0 & 0 & 0 & 0 & 0 \\
\hline \multicolumn{7}{|c|}{ Specific treatment-related $\mathrm{AEs}^{\mathrm{c}}$} \\
\hline \multicolumn{7}{|l|}{$\begin{array}{l}\text { Gastrointestinal } \\
\text { disorders }\end{array}$} \\
\hline Abdomin & 0 & 0 & 0 & 0 & 1 & 0 \\
\hline Constipat & 0 & 0 & 0 & 0 & 1 & 0 \\
\hline Flatulence & 0 & 0 & 1 & 0 & 0 & 0 \\
\hline \multicolumn{7}{|l|}{$\begin{array}{l}\text { Skin and subcutaneous } \\
\text { tissue }\end{array}$} \\
\hline Rash erythematous & 0 & 0 & 0 & 0 & 0 & 1 \\
\hline
\end{tabular}

${ }^{a}$ Includes one volunteer who withdrew and was replaced.

${ }^{\mathrm{b}}$ Data are number of volunteers.

${ }^{c}$ Data are number of AEs.

AE, adverse event; b.i.d., twice daily; q.d., once daily; SPS, sodium polystyrene sulfonate; t.i.d., three times daily

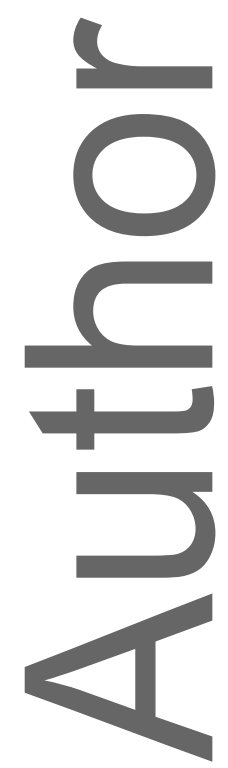




\section{Figure Legends}

A
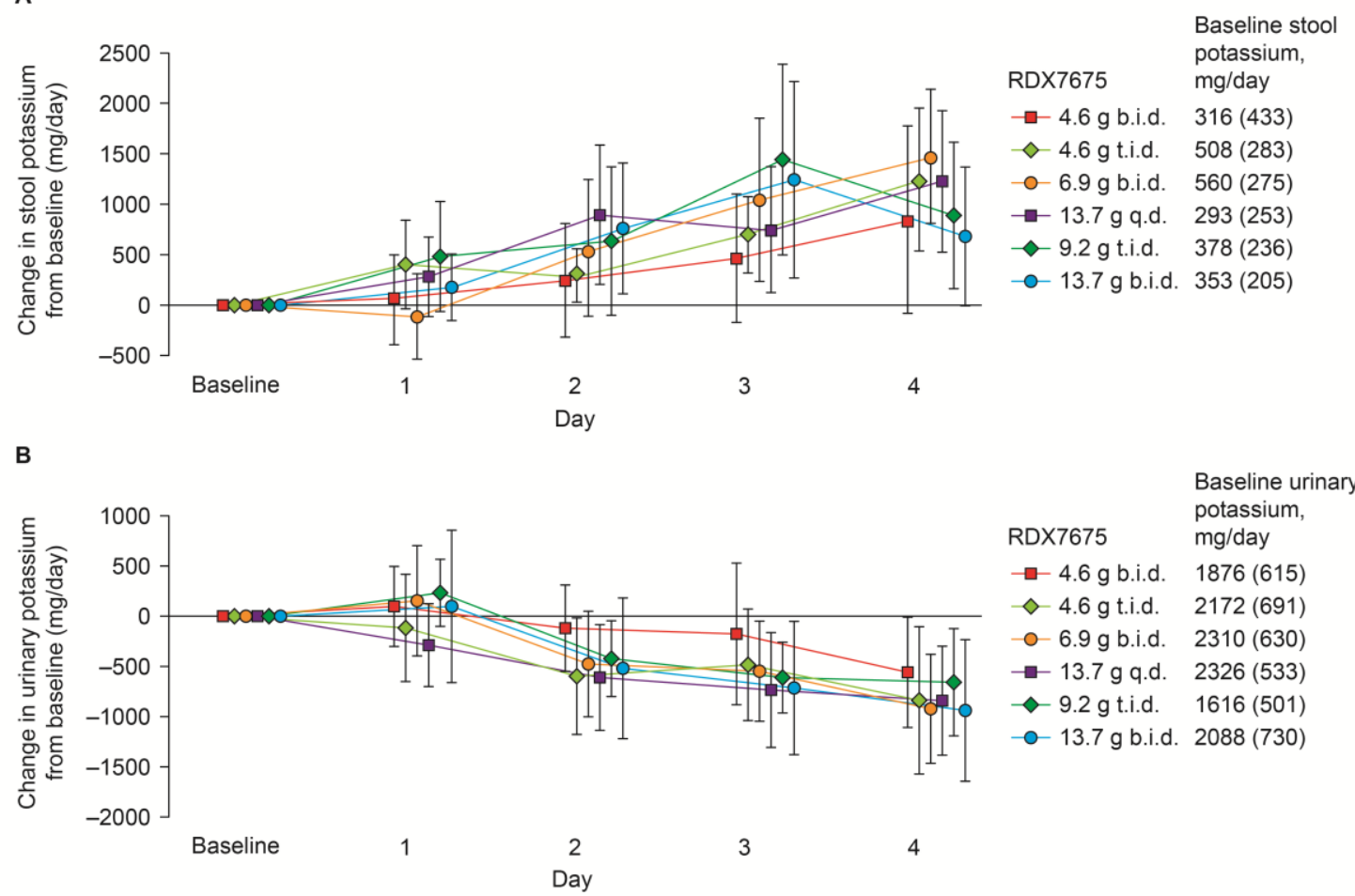

Baseline urinary

RDX7675 potassium

$\because 4.6 \mathrm{~g}$ b.i.d. $\quad 1876(615)$

$\diamond 4.6$ g t.i.d. $2172(691)$

- 6.9 g b.i.d. $2310(630)$

-13.7 g q.d. $2326(533)$

$\checkmark 9.2$ g t.i.d. $1616(501)$

- 13.7 g b.i.d. $2088(730)$

Figure 1. Change in daily potassium excretion from baseline in (A) stool and (B) urine over

4 days in healthy volunteers given RDX7675.

Data are mean \pm standard deviation $(n=12$ for each cohort, except: day 1 urinary potassium,

$4.6 \mathrm{~g}$ b.i.d., $\mathrm{n}=11$; day 1 urinary potassium, $13.7 \mathrm{~g}$ b.i.d., $\mathrm{n}=11$; day 2 stool potassium, $4.6 \mathrm{~g}$

b.i.d., $n=11$ )

Baseline values are mean (standard deviation) over days -2 and -1 .

b.i.d., twice daily; q.d., once daily; t.i.d., three times daily. 